\title{
Portable spectral device for monitoring plant stress conditions
}

\author{
Alexander Smirnov ${ }^{1, *}$, Yuri Proshkin, Alexander Sokolov ${ }^{1}$, and Alexey Dorokhov ${ }^{1}$ \\ ${ }^{1}$ Federal Scientific Agroengineering Center VIM, 109428, 1st Institutsky proezd, 5, Moscow, Russia
}

\begin{abstract}
The non-destructive monitoring systems use for plant stress conditions detecting and evaluating can improve the performed research and working procedures quality significantly. Such systems concepts are based on obtaining of the leaves' reflective characteristics, the spectrum, and the chlorophyll fluorescence induction. By several methods combining for plant stress states detection within a single device, for different crops, it is possible to increase the evaluation accuracy and extend the device functionality. The laboratory prototype of the device for plants' stress states determining was developed and tested on plants of basil garden variety. It was shown that the impact of the stress factor in the form of the UV-C radiation was expressed in the shape change of the fluorescence spectrum, namely, in the fluorescence intensity fall in the long-wave part of the spectrum. Also it was shown that the reflectivity decreased in the green and far red regions of the spectrum. At the spectra registration in the red and IR sections of the spectrum, some noises and interference distortions were detected, which can be filtered out by ways of demodulation and calculation of the trigonometric component of the interference distortion functions.
\end{abstract}

\section{Introduction}

Nowadays, the digital technologies introduction in the processes of information obtaining, processing and analyzing can improve significantly the quality of any performed researches and working procedures. One of such directions is the use of non-destructible monitoring systems for detection and evaluation of stress conditions for the photocultures. This method is based on the chlorophyll fluorescence registration. The fluorescence of the chlorophyll can be used for judging about state of the plant's photosynthetic apparatus and for effectiveness investigating of photochemical reactions associated with functioning of the photosynthetic apparatus, primarily of the photosystem II [1]. The fluorescence induction is a change in the chlorophyll fluorescence intensity, when green leaves are illuminated after keeping the plants in the dark. There is a distinction between fast and slow induction. The duration of the fast fluorescence induction is less than $1 \mathrm{sec}$ starting with the moment of the excitant radiation initiation and up to the maximum level of the fluorescence intensity (Figure 1). Unlike the fast one, the slow induction of chlorophyll fluorescence lasts for several minutes, within which time it passes from its maximum level to its

*Corresponding author: as984788@gmail.com 
stationary value. The measurement method of the chlorophyll fluorescence induction allows assessing the impact of a number of factors on the plants' photosynthetic apparatus. Among those factors, there are a lack of irrigation, an unbalanced mineral nutrition, a chemical contamination, viral/mycotic infections, etc. [2-4].

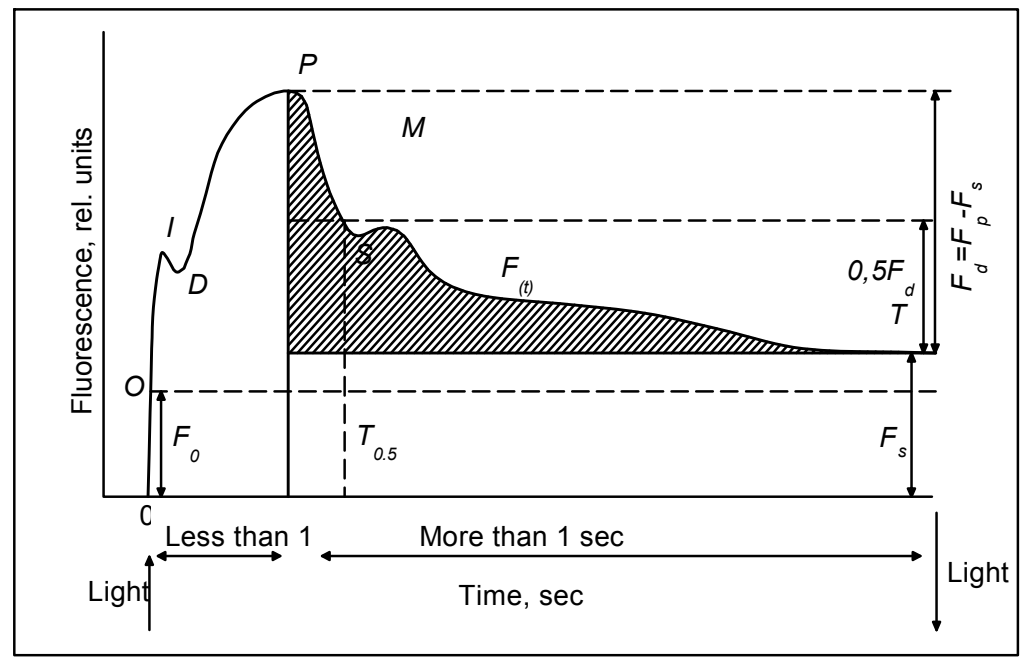

Fig. 1. Typical chlorophyll fluorescence induction curve and its main parameters [5], $F_{0}$ is for zero level of fluorescence intensity; $F_{P}$ for maximum fluorescence intensity at $P ; F_{(t)}$ for current value of fluorescence intensity at a moment $t$ of time; $F_{d}$ for variable part of maximum fluorescence during the slow induction stage; $F_{S}$ for stationary fluorescence level; $T_{0.5}$ for half-fall of fluorescence intensity from its maximum $P$ to stationary level $F_{S}$; $S$ for integral representing area bounded by curve $F_{(t)}$ from above and by straight line $F_{(t)}=F_{S}$ from below.

Also, the vegetation stress states detecting is possible by the way of the fluorescence spectrum determination. [6] The method consists in estimation of the fluorescence radiation spectral composition in the range of $600-750 \mathrm{~nm}$. As a rule, the radiation in the range of $400-500 \mathrm{~nm}$ is taken as the excitation wavelength as the optimal spectrum region of the fluorescence excitation is found at the maximum absorption of a substance, in this case, the chlorophyll [7].

The chlorophyll fluorescence spectrum has two specific peaks: in the red region $680 \ldots 690 \mathrm{~nm}$ and in the far red one $735 \ldots 740 \mathrm{~nm}$ (Figure 2). The ratio of the fluorescence levels $F_{680} / F_{740}$ and $F_{690} / F_{740}$ can be used as an indicator of a chlorophyll content as well as a short-term (at a photosynthetic decline) or a long-term stress indicator $[6,8]$. 


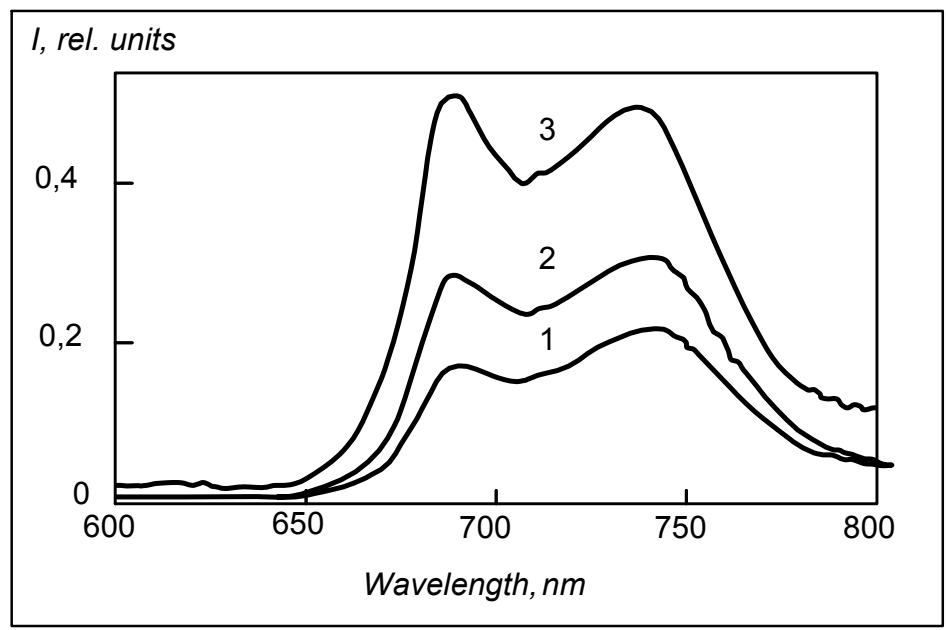

Fig. 2. Fluorescence spectra of cress plants in normal state (1) in comparison with the stressful states (2 and 3 ) caused by humidity excess when watering [9]

With an increase in the chlorophyll concentration, the position of the maximum red fluorescence is shifted to the long-wave region of the spectrum. Also the correlation is observed between the ratio F680/F740 / F690/F740 and the chlorophyll concentrations ratio $a / b[10]$.

Currently, portable devices exist including - among their other functions - the curves construction of the chlorophyll fluorescence induction. The curves allow evaluating the interaction of the photochemical reactions in the photosynthesis light phase and the biochemical reactions effectiveness during the dark phase. As a rule, those devices consist of a source of an excitant radiation in form of a led or a semiconductor laser emitting in the blue region of the spectrum and a photodetector sensitive to the red region of the spectrum. Those devices are quite simple in their design; nevertheless, they do not provide with a fluorescence spectral analysis [11].

For the plants states controlling, some optical methods of spectral analysis of reflected radiation are used, too. The method consists in use of spectrometers or hyperspectral cameras for evaluation of the reflection spectra of green vegetation when it is exposed to visible light in a wide spectral range. As a source of radiation in the portable devices, halogen lamps and/or led combinations are used. For remote sensing, hyperspectral cameras are used and in this case, plants are exposed to the natural sunlight radiation. The remote sensing methods have a number of evident limitations (for example, the night sensing is impossible); besides, they are less accurate than the contact methods.

For assessment of the vegetation state characteristics, special sophisticated parameters are used called vegetation indices that are determined by the difference in their reflectivity in the visible (VIS) and near-infrared (NIR) ranges of the spectrum [12]. The plants states caused by stressful conditions are manifested in a sharp drop in the leaves reflectivity in the far red spectral range. It is known that the chlorophyll absorbs radiation in the blue and red spectral ranges, so healthy leaves have a low backscatter coefficient at the chlorophyll maximum absorption. Changes in the chlorophyll content in plant leaves can be determined by measurement of the reflectivity factors in the green, red and blue regions of the spectrum. The stress reduces the plants' reflectivity in the green region of the spectrum and simultaneously it increases the reflectivity in the blue and red regions. Also, the steepness is reduced of the reflectivity transition from the red region of the spectrum to the far red one [13]. 
Thus, by combining several methods for plant stress states determining in a single device, it is possible to increase the measuring accuracy and extend the functionality of its use for different crops.

\section{Materials and Methods}

The laboratory prototype of the device able to determine plant stress states has been developed (Figure 3). The device consists of a Y-shaped optical probe of reflection/backscattering. The probe contains a bundle of seventeen tightly packed optical fibers of the same diameter of 200 microns in the UV-VIS range $(250-800 \mathrm{~nm})$. As the radiation source in the experiments, the led lasers were used with their radiation maxima at $380,405,460$ and $640 \mathrm{~nm}$ and the halogen lamp with continuous radiation in the range of $400-800 \mathrm{~nm}$. Both the reflected and fluorescent radiation was filtered with use of glass optical filters cutting off the short-wave range of the spectrum. The device for spectrometric information reading (spectrometer) was implemented on the basis of the following elements: the programmable gate array Altera Cyclone EP1C3T100C8N, the diffraction grating $(600 \mathrm{bars} / \mathrm{mm})$ and the linear array photoelectric receptor Toshiba TCD1304. The integration time made 5-100ms.

Our laboratory tests of the developed device were carried out on plants of basil gardenvariety (Ocīmum basilīicum) of the Lemon's sort. The plants were grown in cameras with the same microclimatic conditions but some plants were exposed to the stress factors in the form of the UV-C radiation. Under analysis, there were the fluorescence spectrum of the chlorophyll at excitement by various radiation sources and the reverse reflection spectrum at irradiation from the halogen lamp.

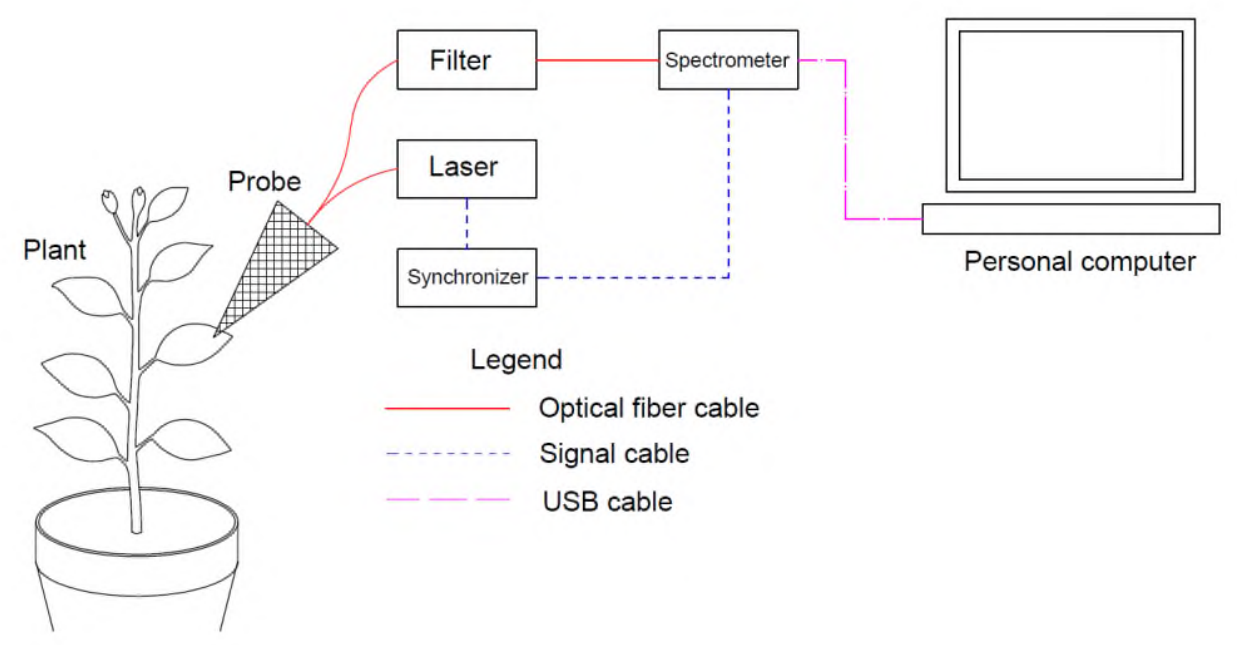

Fig. 3. Scheme of spectrum registration of chlorophyll fluorescence

\section{Results}

The analysis of the spectra shows that when excited by the laser with the wavelength of $380 \mathrm{~nm}$, the second order of diffraction falls on the recorded fluorescence spectrum, which leads to its distortion. Use of a cut-off filter can reduce or eliminate this negative factor. At excitement by the laser with a wavelength of $640 \mathrm{~nm}$, the first (basic) order of the 
diffraction is located close to the recorded fluorescence spectrum, which also introduces a distortion. Hence, in order to excite the fluorescence of the chlorophyll, the best option is use of lasers with wavelengths in the range of $405 \div 470 \mathrm{~nm}$. Based on the $460 \mathrm{~nm}$ laser, the fluorescence spectra of the basil chlorophyll were obtained in plants' normal state and in stress caused by the UV-C irradiation during the vegetation growth (Figure 4).

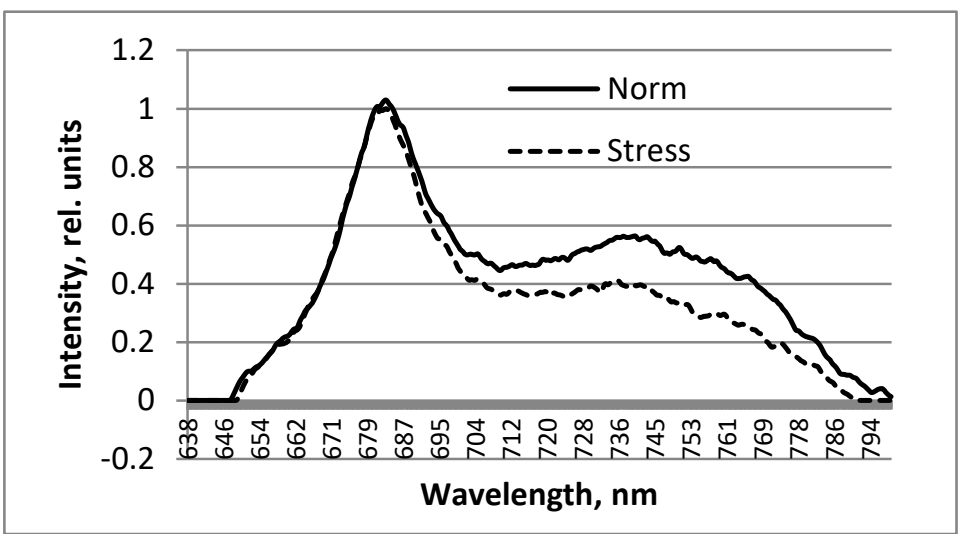

Fig. 4. Diagrams of fluorescence spectra of basil chlorophyll in both normal and stressful conditions

Figure 5 shows the reflection spectrum of basil leaves in normal and stress conditions. At the spectrum recording, the leaves were illuminated by the halogen lamp.

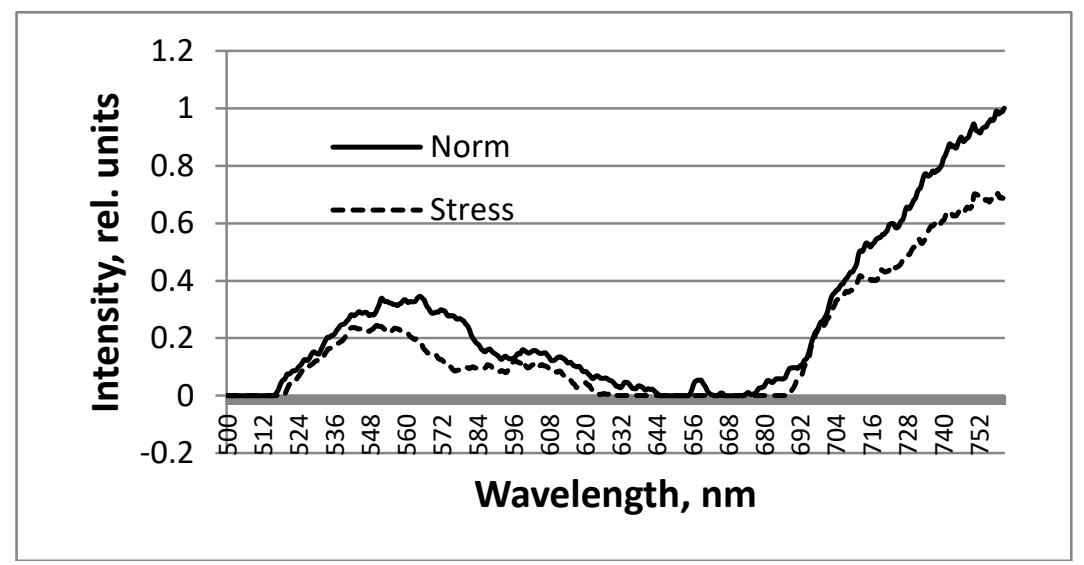

Fig. 5. Plant leaf reflectance spectrum

\section{Discussion}

It follows from the Figure 4 that the influence of the stress factor is manifested in the shape change of the fluorescence spectrum. The ratio of the maxima in the fluorescence spectrum depends on the aggregation degree of the chlorophyll forms and the radiation re-absorption. In this case, the stress is seen in the fluorescence intensity decrease in the long-wave part of the spectrum, which is explained by fluctuations in the activity of the photosystem II [9].

On the diagram (Figure 5), the reflectivity specific peak is visible in the green and far red regions of the spectrum. A stress manifests itself in the reflectivity fall in the green and far red regions of the spectrum; in addition, the curve steepness is reduced when moving from the red to the far red region of the spectrum. 
At the spectra recording, some noise and interference were detected in the red and IR sections of the spectrum; meanwhile the same was hardly observed in the green section of the spectrum. (Figures 4 and 5). It is known that many modern spectrometers distort the recorded data when receiving radiation spectra; this becomes a serious problem when studying and detecting weak fluorescence spectra such as the spectrum of the chlorophyll fluorescence. This phenomenon is well observed, when the light source has a wide and continuous spectrum.

The interference is caused by the sequential reflection of light from the upper and lower edges of a film; it results in a co-directional and reflected wave, which is superimposed on the original wave but with a certain delay. The interference runs separately for each wavelength; therefore, it makes a significant impact on all measurements, even on the mercury peaks; so the measurements are interfered and expressed in incorrect values. Unfortunately, notwithstanding that the distortions are clearly visible on a continuous spectrum, they are not seen on narrow peaks; so it is necessary to demodulate the entire spectrum diagram.

The issues of signal processing and adaptive noises detection in physical processes have been considered by many authors [14-16]. The interference was described by the formula:

$$
I=I_{0}+k \cdot I_{0} \cdot \cos (w x+\phi)
$$

where $I_{0}$ is for original intensity; $I$ for resulted intensity after interference; $x$ for wave length, $\phi$ for some phase shift; $k$ for coefficient depending on the reflectivity factor of the protective film (it is $<1$ because the reflected wave is much weaker than the original one); and $w$ for distortions caused by the film depending on its thickness $L$.

As it seems impossible to determine $L$ with nanometer accuracy, it is necessary to determine the value of $w$ by trial-and-error values selection. In the course of the work, for the three central peaks, a peaks shift of relative periodicity was revealed. The reason for this is that $w$ is not directly proportional to $L$ as the reflecting/transmitting properties of the film differ in different parts of the spectrum; so it is necessary to construct the formula like this:

$$
I=I_{0}+k \cdot I_{0} \cdot \cos (f(x))
$$

where $f(x)$ is for some function of $x$.

In order to identify this function, it is necessary to implement the following operations:

1. to obtain measurements on a reference spectrometer so that the results would be comparable; the light source spectrum must be broadband,

2. to compare the true spectrum with the resulted one in order to determine all the extremes on the diagram,

3. to restore the function $f(x)$ based on the extremes.

The function $f(x)$ will be the same for all the measurements on this spectrometer. Now knowing $f(x)$, one can deduce that the true spectrum is equal to $I /(1+k \cdot \cos (f(x))$.

As an alternative to the demodulation and the calculation of the trigonometric component of the interference distortion functions, another method can be used. It is based on the straight line inclination angle determination with use of the inflection points. In order to do this, it is needed to calculate the average values between the interpolation peaks. The result can be represented as a diagram. If we know the background noise value and the sensitivity of the device spectral scale, it is possible to get correction factors that would allow constructing the measured spectrum diagram with a sufficient accuracy.

The second problem consists in the nonlinear sensitivity of both the photodetector and the diffraction grating for different parts of the spectrum. Another problem is the displacement of the wavelength, absorption and reflection of part of the radiation at light passage through both the protective film of the photodetector and the optical fiber. The 
detected noise can be reduced by increasing of the impinging light emission power and integration times; or it can be done by programming. In the subsequent studies, consideration will be given to the methods of spectral signals processing for the oscillation filtering and the nonlinear sensitivity eliminating of the photodetector being part of the spectrometer; In addition, an algorithm for plant stress states determination will be developed.

\section{Conclusions}

The laboratory prototype has been developed of the combined portable device for plants' stress states determination based on recording of the reflective parameters of leaves, their spectrum and the chlorophyll fluorescence induction.

The tests have shown that when the procedure is aimed at the chlorophyll fluorescence spectrum registration, the best option for its excitation is lasers use with wavelengths in the range of $405 \div 470 \mathrm{~nm}$.

The impact of the stress factor in the form of the UV-C radiation on basil plants is observed in a shape change in the chlorophyll fluorescence spectrum and a decrease in reflectivity in the green and far red regions of the spectrum.

The comprehensive analysis of both the chlorophyll fluorescence spectra and the radiation reflected from leaves at the same time can improve the accuracy of the plants' stress conditions determination.

\section{References}

1. J. M. Banks, Environmental and Experimental Botany, 155, 118-127 (2018) https://doi.org/10.1016/j.envexpbot.2018.06.022

2. Ch. Srinivasarao, A. K. Shanker, S. Kundu, S. Reddy, Journal of Photochemistry and $\begin{array}{lllll}\text { Photobiology } & \text { B: } & \text { Biology, } & \text { 160, } & \text { 86-95 }\end{array}$ https://doi.org/10.1016/j.jphotobiol.2016.03.052

3. P. Dąbrowski, A. H. Baczewska, B. Pawluśkiewicz, M. Paunov, V. Alexantrov, V. Goltsev, M. H. Kalaji, Journal of Photochemistry and Photobiology B: Biology, 157, 22-31 (2016) https://doi.org/10.1016/j.jphotobiol.2016.02.001

4. L. Guidi, E. Degl'Innocenti, G. Carmassi, D. Massa, A. Pardossi, Environmental and $\begin{array}{llll}\text { Experimental } \quad \text { Botany, 57-63 } & \text { (2011) }\end{array}$ https://doi.org/10.1016/j.envexpbot.2010.09.017

5. T. V. Nesterenko, A. A. Tikhomirov, V. N. Shikhov, Biofizika, 57, 614-620 (2012)

6. V. Lysenko, Y. Guo, A. Kosolapov, E. Usova, T. Varduny, V. Krasnov, Information $\begin{array}{lllll}\text { Processing in } & \text { Agriculture, 204-211 }\end{array}$ https://doi.org/10.1016/j.inpa.2019.09.009

7. M. L. Belov, D. V. Vladimirsky, V. A. Gorodnichev, B. V. Strelkov, Mechanical Engineering and Computer Technologies, 1, (2014) DOI: 10.7463/0114.0636811

8. V. Lysenko, T. Varduny, E. Simonovich, O. Chugueva, V. Chokheli, M. Sereda, et al., Biochem Biotechnol; 10(4), 234-40 https://doi.org/10.3844/ajbbsp.2014.234.240.

9. M. L. Belov, O. A. Bullo, Yu. V. Fedotov, V. A. Gorodnichev, Instrument making, 2, 71-82 (2015)

10. N. Vilfan, C. an der Tol, O. Muller, et al., Remote Sensing of Environment, 186, 596615 (2016) DOI: 10.1016/j.rse.2016.09.017 
11. A. A. Fernandez-Jaramillo, R. de J. Romero-Troncoso, C. Duarte-Galvan, I. TorresPacheco, R. G. Guevara-Gonzalez, L. Miguel Contreras-Medina, G. Herrera-Ruiz, J. R. Millan-Almaraz, Measurement, 75, 12-22 https://doi.org/10.1016/j.measurement.2015.08.005

12. E. E. da Silva, F. H. R. Baio, Larissa Pereira Ribeiro Teodoro, Carlos Antonio da Silva Junior, Raisa Saraiva Borges, Paulo Eduardo Teodoro, Remote Sensing Applications: $\begin{array}{lllll}\text { Society and } \quad \text { Environment, } & 100318 & \text { (2020) }\end{array}$ https://doi.org/10.1016/j.rsase.2020.100318

13. M. L. Belov, Y. S. Fesenko, V. A. Gorodnichev, A. V. Kuvshinov, Radio Engineering, 3, 1-17 (2016) (In Russ.) DOI: 10.7463/rdopt.0316.0840843

14. F. Z. El Bahi, H. Ghennioui, M. Zouak, Physical Communication, 33, 142-150 (2019) https://doi.org/10.1016/j.phycom.2018.12.022

15. C.-C. Tseng, S.-L. Lee, Signal Processing, 137, 80-97 (2017) https://doi.org/10.1016/j.sigpro.2017.01.015

16. A. Borovich, A. Petrovsky, Speech Technologies, 3-4, 12-29 (2013) 